\title{
Gammoids, Pseudomodularity and Flatness Degree
}

\author{
Jorge Alberto Olarte \\ Departmento de Matemáticas \\ Universidad de Los Andes \\ Bogotá, Colombia \\ ja.olarte1299@uniandes.edu.co
}

\begin{abstract}
Submitted: Sep 8, 2014; Accepted: Feb 14, 2015; Published: Mar 6, 2015
Mathematics Subject Classifications: 05B35, 05C89
\end{abstract}

\begin{abstract}
We introduce the concept of flatness degree for matroids, as a generalization of submodularity. This represents weaker variations of the concept of flatness which characterize strict gammoids for finite matroids. We prove that having flatness degree 3 , which is the smallest non-trivial flatness degree, implies pseudomodularity on the lattice of flats of the matroid. We show however an example of a gammoid for which the converse is not true. We also show examples of gammoids with each possible flatness degree. All of this examples show that pseudomodular gammoids are not necessarily strict.
\end{abstract}

\section{Introduction}

Hrushovski introduces the concept of a matroid being flat in [8] in order to prove the existence of a non trivial strongly minimal set that does not interpret an infinite field. In [5], Evans shows that for finite matroids the notion of flatness characterizes the matroids known as strict gammoids, a class of matroids that arises from directed graphs. Strict gammoids are also known as cotransversal matroids, as Ingleton and Piff showed that they are precisely the duals of transversal matroids [11]. Actually, in [12] Mason gives the exact dual analog of flatness as a characterization of transversal matroids. The restrictions of a strict gammoid, are known as simply gammoids and they form a complete class of matroids that has been widely studied $[3,5,9,11,12,13,14]$.

In [5], Evans shows that strict gammoids have a pseudomodular lattice of flats. The notion of pseudomodularity was first studied by Dress and Lovász in [4] as a necessary condition for full algebraic matroids and was formally defined in [1] by Björner and Lovász. Evans asks whether a gammoid which is pseudomodular is necessarily a strict gammoid. We answer negatively by showing that a strictly weaker condition than flatness, having 
flatness degree at least 3 is enough for pseudomodularity. We then construct a gammoid showing that the converse is not true, that is being pseudomodular and having flatness degree 2. We construct of each possible flatness degree. All of these are counter-examples to Evans' question. We get an infinite chain of increasingly stronger properties, from pseudomodularity to flatness, all of them in terms of the lattice of flats.

We review basic results about matroids and the gammoid class in Section 2. We define and discuss pseudomodularity in Section 3 and we connect it with the notion of flatness. In Section 4 we define the flatness degree and construct examples of gammoids that attain each of the possible values for flatness degree. Finally we propose some problems in Section 5.

\section{Matroid theory background}

In this section we review basic matroid theory in order to fix notation. This can also be helpful for model theorists who may not be familiar with matroids. Proofs and further insight can be found in [14] and, for the specific gammoid class, in $[2,3]$. Throughout the paper we consider only finite matroids although most of the results can be extended to any infinite but finitary (finite rank) combinatorial pregeometry. A matroid $M$ consists of a (finite) set $N$ and a function $r: \mathcal{P}(N) \rightarrow \mathbb{Z}$ called rank that satisfy the following conditions

(R1) If $A \subseteq N$ then $0 \leqslant r(A) \leqslant|A|$

(R2) If $A \subseteq B \subseteq N$ then $r(A) \leqslant r(B)$

(R3) If $A \subseteq N$ and $B \subseteq N$ then $r(A)+r(B) \geqslant r(A \cup B)+r(A \cap B)$

The last condition is called submodularity. The rank of a matroid is $r(N)$. A subset $I$ of $N$ is called independent if $r(I)=|I|$. A subset $B$ of $N$ is called a basis if it is independent and $r(B)=r(N)$. A subset $C$ of $N$ is a circuit if it is a minimal dependent set. That is, for every $e \in C, r(C)=r(C-\{e\})=|C|-1$. A subset $F$ of $N$ is called a flat if for every $n \in N-F$ we have $r(F \cup\{n\})>r(F)$. We will write $\mathcal{F}=\mathcal{F}(M)$ as the set of flats of the matroid $M$. A flat of rank $r(N)-1$ is called a hyperplane. When the equality in (R3) is met whenever $A$ and $B$ are flats, we say that $M$ is modular. We can define the closure operator $c l: \mathcal{P}(N) \rightarrow \mathcal{P}(N)$ as $c l(A):=\min (\{F \in \mathcal{F} \mid A \subseteq F\})$ or, equivalently $\operatorname{cl}(A)=\max (\{A \subseteq F \subseteq N \mid r(A)=r(F)\})$.

Note that the rank of $A$ tells us the maximum cardinality of an independent subset of $A$. Then the matroid is determined by the set of independent subsets of $N$. Also, the rank of a flat $F$ is the length of a maximal chain of flats such that $F_{0} \subset F_{1} \subset \cdots \subset F_{r(F)}=F$. As the rank of any set is the rank of its closure, which is a flat, the matroid is also determined by the set of flats. In fact, matroids can be cryptomorphically defined by any of the objects defined above. We choose to use the rank function for the definition, because of the importance of submodularity in this paper. So sometimes we will refer to 
a matroid by giving its set of independent sets or the set of flats rather than giving the pair $(N, r)$.

Given a matroid $M$ on the set $N$, we can naturally define matroids on subsets of $N$. More precisely, for $A \subseteq N$, we define the restriction of $A$ as the matroid $M \backslash A$ on the set $N-A$ with rank function $r_{M \backslash A}$ defined as the rank function restricted to $\mathcal{P}(N-A)$. We can also define the contraction of $A$ as the matroid $M / A$ which also has ground set $N-A$ but with rank function $r_{M / A}(B):=r(B \cup A)-r(A)$ for any $B \subseteq N-A$. The operations of restricting and contracting a matroid commute, that is $(M \backslash A) / B=(M / B)-A$. Any matroid constructed this way is called a minor of $M$.

Finally we can define the dual $M^{*}$ of $M$, acting on the same set $N$ but with rank function $r^{*}(A):=|A|+r(N-A)-r(N)$. It can be easily verified that the bases of $M^{*}$ are precisely the complements of the bases of $M$. Thus, $\left(M^{*}\right)^{*}=M$ and hence the term dual. It can be shown that if $A \subseteq N$ then $(M \backslash A)^{*}=M^{*} / A$. Note that this implies $M^{*} \backslash A=(M / A)^{*}$. So in this sense we can say that the restriction and the contraction are dual. There are many more objects that can be said to be dual in some sense. An element $n \in N$ is said to be a loop if $r(n)=0$. An element $n \in N$ is a coloop if it is a loop in $M^{*}$ or, equivalently, if for all $A \subseteq N A$ is independent if and only if $A \cup\{n\}$ is independent. A subset $S$ of $N$ is called cyclic if it satisfies that for every $e \in S, r(S-\{e\})=r(S)$. In other words, $S$ is cyclic if $M \backslash(N-S)$ does not have coloops. Note that all circuits are cyclic. It is easy to verify that $S$ is cyclic if and only if $N-S$ is a flat.

We now turn our attention to the class of gammoids, starting with transversal matroids. Given $\mathcal{A}=\left(A_{1}, \ldots, A_{k}\right)$ subsets of a set $N$, and a subset $I$ of $N$, a matching of $I$ is an injective function from $I$ to $\mathcal{A}$ such that for every $n \in I$, we have $n \in f(n)$. The set of subsets of $N$ that have a matching form the independent sets of a matroid $M$. The set $\mathcal{A}$ is called a presentation of $M$. A matroid that can be constructed this way is called transversal. Different presentations may produce the same matroid. It is obvious from the definition that the rank of $M$ is at most $k$, however, one can always give presentations such that $k$ is exactly the rank of $M$.

Now let $\Gamma=(N, E)$ be a directed graph where $N$ is the set of vertices and $E$ is the set of edges. Given $u, v \in N$, a path $P$ from $u$ to $v$ is a sequence of vertices $\left(n_{1}, \ldots, n_{t}\right)$ such that $n_{1}=u, n_{t}=v$ and for every $i \leqslant t-1,\left(n_{i}, n_{i+1}\right) \in E$. Given two sets $A, B \subseteq E$, a linking $\Theta$ from $A$ to $B$ is a collection of paths such that

- $|\Theta|=|A|$

- The paths are pairwise disjoint

- Each path starts in a vertex belonging to $A$ and ends in a vertex of $B$

Fixing a sets $N_{1}, N_{2} \subseteq N$, the sets $I \subseteq N_{1}$ such that there is a linking from $I$ to $N_{2}$ are the independent sets of a matroid $M$ on the set $N_{1}$. A matroid that can be constructed this way is called a gammoid. If $N_{1}=N$ we say it is a strict gammoid. A gammoid can have different directed graphs representing it.

The gammoid class is closed under minors and duals. Strict gammoids and transversal matroids are known to be dual [11]. It is clear from the definition that transversal matroids 
are closed under restriction, however they are not closed under contraction. Dually, strict gammoids are closed under contraction but not closed under restriction. Every gammoid is the restriction of a strict gammoid. Then every gammoid is the contraction of a transversal matroid.

Example 1. Let $\Gamma$ be the graph shown in Figure 1. By letting $N_{1}=\{1,2,3,4,5,6\}$ and $N_{2}=\{2,4,6, x\}$ we get a gammoid $M$ of rank 4 . This gammoid is also a transversal matroid, as $\{\{1,2\},\{3,4\},\{5,6\},\{1,3,5\}\}$. is a presentation of $M$. However it will be shown in Section 3 that $M$ is not a strict gammoid (this does not follow from the fact that $x \notin N_{1}$, as there may be a different directed graph representing $M$ with all of its vertices).

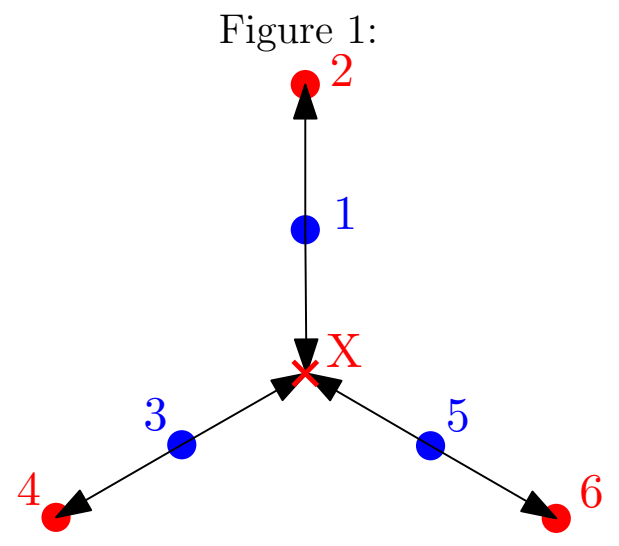

\section{3-Flatness and Pseudomodularity}

There have been many characterizations for transversal matroids and strict gammoids, most of them in terms of flats and specially cyclic flats. However, the characterization for strict gammoids which is more appropriate for our purposes is Theorem 3.2. Mason first showed the analogue for transversal matroids in [12]. However, it is specifically stated and shown by Evans in [5].

Let $M$ be a matroid. Recall that we write $\mathcal{F}$ for the set of flats of $M$. Let $\mathcal{C}=\left\{F_{i} \in\right.$ $\mathcal{F} \mid i \in I\}$ be a collection of flats. If $\emptyset \neq S \subseteq I$ let $F_{S}=\bigcap_{i \in S} F_{i}$ and $F_{\emptyset}=\bigcup_{i \in I} F_{i}$. We define the $\Delta$ function as

$$
\Delta(\mathcal{C})=\sum_{S \subseteq I}(-1)^{|S|} r\left(F_{s}\right)
$$

For such $\mathcal{C}$ we want to admit repetitions even though they are trivial for calculating $\Delta(\mathcal{C})$, thus letting $\mathcal{C}$ be a multiset. We want to do this, because we are going to manipulate the elements in $\mathcal{C}$ and want to maintain the cardinality of $\mathcal{C}$ constant. The $\Delta$ function will help us define the concept of flatness given by Hrushovski in [8].

Definition 3.1. We say $M$ is totally flat if $\forall \mathcal{C} \subseteq \mathcal{F}$ finite, $\Delta(\mathcal{C}) \leqslant 0$ 
Theorem 3.2. A finite matroid $M$ is a strict gammoid if and only if it is totally flat.

Consider Example 1. Let $\mathcal{C}=\{\{1,2,3,4\},\{1,2,5,6\},\{3,4,5,6\}\}$ we have

$$
\begin{aligned}
\Delta(\mathcal{C}) & =r(\{1,2,3,4,5,6\})-r(\{1,2,3,4\})-r(\{1,2,5,6\})-r(\{3,4,5,6\}) \\
& +r(\{1,2\})+r(\{3,4\})+r(\{5,6\}) \\
& =4-3-3-3+2+2+2=1
\end{aligned}
$$

so $M$ is not totally flat. Hence, it is not a strict gammoid. It is actually the minimal gammoid that is not strict gammoid, in terms of rank and size.

Definition 3.3. A matroid $M$ is $n$-flat, if and only if $\forall \mathcal{C} \subseteq \mathcal{F}$ such that $|\mathcal{C}| \leqslant n, \Delta(\mathcal{C}) \leqslant 0$.

We used the term $n$-flat for $|\mathcal{C}| \leqslant n$ instead of $|\mathcal{C}|=n$ because once $\Delta(C)>0$ it is easy to generate collections of sets of larger cardinality than $\mathcal{C}$ by adding any subflat of elements of $\mathcal{C}$ (see Proposition 4.2). Note that 2-flat is equivalent to submodularity, so all matroids are trivially 2 -flat. The first non-trivial property would be 3 -flat, and it happens to imply several important properties.

Now let $A, B \in \mathcal{F}$. We write $r(A / B):=r(A \cup B)-r(B)$, which is the rank of $A$ when contracting $B$. Suppose there exists $B_{0} \in \mathcal{F}$ such that for every flat $B_{1} \subseteq B$, $r\left(A / B_{1}\right)=r(A / B)$ if and only if $B_{0} \subseteq B_{1} . B_{0}$ is called the pseudointersection of $A$ and $B$. Note that the pseudointersection is not symmetric, that is, the pseudointersection of $A$ and $B$ can be different from the pseudointersection of $B$ and $A$. Further, not even the existence of pseudointersection is symmetric. Example of these may be seen in [1]. If the pseudointersection of $A$ and $B$ exists, we write $A \triangleleft B$. If it does not exist, we write $A \not B$.

Definition 3.4. A matroid $M$ is pseudomodular if for every $A, B \in \mathcal{F}$ we have $A \triangleleft B$

The concepts of pseudointersection and pseudomodularity are phrased in terms of the lattice of flats of a matroid and can be extended for any lattice as originally defined in [1]. It is not difficult to see that pseudomodularity may be restated as follows: for every $A, B, C \in \mathcal{F}$ if $r(A / B)=r(A / C)=r(A / B \cup C)$ then $r(A / B \cap C)=r(A / B)$. Consider again Example 1. If $A=\{1,2\}, B=\{3,4,5,6\}, B_{1}=\{3,4\}$ and $B_{2}=\{5,6\}$ we have $r(A / B)=r\left(A / B_{1}\right)=r\left(A / B_{2}\right)=1$. But $B_{1} \cap B_{2}=\emptyset$, so $r(A / \emptyset)=r(A)=2$. Then $A \backslash B$ and so $M$ is not pseudomodular. If we consider the strict gammoid generated by $\Gamma$, say $M_{0}, X$ would be in the closure of $B, B_{1}$ and $B_{2}$, so $B_{1} \cap B_{2}=\{X\}$ and we would have $r(A /\{X\})=1 . M_{0}$ is actually modular, as Evans shows in [5] that strict gammoids are pseudomodular. We show Theorem 3.6 as a generalization of that result. Before proving Theorem 3.6 we need the following lemma

Lemma 3.5. Let $M$ be a matroid which is not pseudomodular. Then there exists $B \in \mathcal{F}$ and $A \subseteq E$ such that $r(A / B)=1$ and $A \not B$.

Proof. If $M$ is not pseudomodular, then there exist $A, B \in \mathcal{F}$ such that $A \backslash B$. This means there are flats $B_{1}, B_{2} \subseteq B$ such that $r(A / B)=r\left(A / B_{1}\right)=r\left(A / B_{2}\right)<r\left(A / B_{1} \cap\right.$ 
$\left.B_{2}\right)$. We now prove the lemma by induction on $r(A / B)$. If $r(A / B)=1$ then we have the desired result. Now let $r(A / B)=n$ and suppose the lemma is true whenever there are $B^{\prime} \in \mathcal{F}, A^{\prime} \subseteq E$ such that $A^{\prime} \not B^{\prime}$ and $r\left(A^{\prime} / B^{\prime}\right)=n-1$. Let $x \in A-B$ and consider the set

$$
C=\left(\operatorname{cl}\left(B_{1} \cup\{x\}\right) \cap \operatorname{cl}\left(B_{1} \cup\{x\}\right)\right)-\left(B_{1} \cap B_{2}\right)
$$

As $C \subseteq \operatorname{cl}\left(B_{1} \cup\{x\}\right), r\left(C \cup B_{1}\right)=r\left(B_{1}\right)+1$ and so $r\left(C / B_{1}\right)=1$. In the same way $r\left(C / B_{2}\right)=1$. As $x \in C-B$ then $r(C / B) \geqslant 1$ and as $B_{1} \subseteq B$ then $r(C / B) \leqslant r\left(C / B_{1}\right)=$ 1 , so $r(C / B)=1$. If $r\left(C / B_{1} \cap B_{2}\right)>1$ then $C \wedge B$ and we have what we want. Suppose now $r\left(C / B_{1} \cap B_{2}\right)=1$. Note that $C \cup\left(B_{1} \cap B_{2}\right)=\operatorname{cl}\left(B_{1} \cup\{x\}\right) \cap \operatorname{cl}\left(B_{2} \cup\{x\}\right)$. Let $B^{\prime}=\operatorname{cl}(B \cup\{x\}), B_{i}^{\prime}=\operatorname{cl}\left(B_{i} \cup\{x\}\right)$ for $i \in\{1,2\}$. As $x \in A, c l\left(A \cup B^{\prime}\right)=\operatorname{cl}(A \cup B)$. Then

$$
\begin{aligned}
r\left(A / B^{\prime}\right) & =r\left(A \cup B^{\prime}\right)-r\left(B^{\prime}\right) \\
& =r(A \cup B)-r(B)-\left(r\left(B^{\prime}\right)-r(B)\right) \\
& =r(A / B)-r\left(B^{\prime} / B\right)=n-1 .
\end{aligned}
$$

In the same way $r\left(A / B_{1}^{\prime}\right)=r\left(A / B_{2}^{\prime}\right)=n-1$. As $B_{1}^{\prime} \cap B_{2}^{\prime}=C \cup\left(B_{1} \cap B_{2}\right)$, we have:

$$
\begin{aligned}
r\left(A / B_{1}^{\prime} \cap B_{2}^{\prime}\right) & =r\left(A \cup\left(B_{1}^{\prime} \cap B_{2}^{\prime}\right)\right)-r\left(B_{1}^{\prime} \cap B_{2}^{\prime}\right) \\
& =r\left(A \cup\left(B_{1}^{\prime} \cap B_{2}^{\prime}\right)\right)-r\left(B_{1} \cap B_{2}\right) \\
& -\left(r\left(B_{1}^{\prime} \cap B_{2}^{\prime}\right)-r\left(B_{1} \cap B_{2}\right)\right) \\
& =r\left(A / B_{1} \cap B_{2}\right)-r\left(B_{1}^{\prime} \cap B_{2}^{\prime} / B_{1} \cap B_{2}\right) \\
& =r\left(A / B_{1} \cap B_{2}\right)-r\left(C / B_{1} \cap B_{2}\right) \\
& =r\left(A / B_{1} \cap B_{2}\right)-1>n-1 .
\end{aligned}
$$

Then $A \backslash B^{\prime}, r\left(A / B^{\prime}\right)=n-1$ and by the induction hypothesis the lemma is true.

Now we can prove the main result of the section

Theorem 3.6. Let $M$ be a 3-flat matroid. Then $M$ is pseudomodular.

Proof. Suppose $M$ is not pseudomodular. We will prove that it is not 3-flat. By lemma 3.5 there are $A, B \in \mathcal{F}$ such that $A \backslash B$ and $r(A / B)=1$. So there are flats $B_{1}, B_{2} \subseteq B$ such that $r\left(A / B_{1}\right)=\left(A / B_{2}\right)=1<r\left(A / B_{1} \cap B_{2}\right)$. Let $F_{1}=\operatorname{cl}\left(A \cup B_{1}\right), F_{2}=\operatorname{cl}\left(A \cup B_{2}\right)$ and $F_{3}=B$ and consider $\mathcal{C}=\left\{F_{1}, F_{2}, F_{3}\right\}$. We have

- $F_{\emptyset} \subseteq \operatorname{cl}(B \cup A)$. Then $r\left(F_{\emptyset}\right)=r(B \cup A)$

- $F_{\{1,2\}}=\operatorname{cl}\left(A \cup B_{1}\right) \cap \operatorname{cl}\left(A \cup B_{2}\right) \supseteq\left(B_{1} \cap B_{2}\right) \cup A$. Then $r\left(F_{\{1,2\}}\right) \geqslant r\left(\left(B_{1} \cap B_{2}\right) \cup A\right)$

- $F_{\{1,3\}}=\operatorname{cl}\left(A \cup B_{1}\right) \cap B=B_{1}$ as $c l\left(A \cup B_{1}\right)-B_{1} \subseteq E-B$. Then $r\left(F_{\{1,3\}}\right)=r\left(B_{1}\right)$

- $F_{\{2,3\}}=\operatorname{cl}\left(A \cup B_{2}\right) \cap B=B_{2}$ as $c l\left(A \cup B_{2}\right)-B_{2} \subseteq E-B$. Then $r\left(F_{\{2,3\}}\right)=r\left(B_{2}\right)$

- $F_{\{1,2,3\}}=B_{1} \cap B_{2}$. Then $F_{\{1,2,3\}}=r\left(B_{1} \cap B_{2}\right)$ 
Then

$$
\begin{aligned}
\Delta(C) & =r\left(F_{\emptyset}\right)-r\left(F_{1}\right)-r\left(F_{2}\right)-r\left(F_{3}\right)+r\left(F_{\{1,2\}}\right) \\
& +r\left(F_{\{1,3\}}\right)+r\left(F_{\{2,3\}}\right)-r\left(F_{\{1,2,3\}}\right) \\
& \geqslant r(B \cup A)-r(B)-r\left(B_{1} \cup A\right)-r\left(B_{2} \cup A\right) \\
& +r\left(\left(B_{1} \cap B_{2}\right) \cup A\right)+r\left(B_{1}\right)+r\left(B_{2}\right)-r\left(B_{1} \cap B_{2}\right) \\
& =(r(B \cup A)-r(B))-\left(r\left(B_{1} \cup A\right)-r\left(B_{1}\right)\right) \\
& -\left(r\left(B_{2} \cup A\right)-r\left(B_{2}\right)\right)+\left(r\left(\left(B_{1} \cap B_{2}\right) \cup A\right)-r\left(B_{1} \cap B_{2}\right)\right) \\
& =r(A / B)-r\left(A / B_{1}\right)-r\left(A / B_{2}\right)+r\left(A / B_{1} \cap B_{2}\right) \\
& =1-1-1+r\left(A / B_{1} \cap B_{2}\right) \\
& =r\left(A / B_{1} \cap B_{2}\right)-1 \\
& >0 .
\end{aligned}
$$

Note that the lemma was used to ensure $F_{\{1,3\}}=B_{1}$ and $F_{\{2,3\}}=B_{2}$. If $r\left(A / B_{1}\right)>1$, then we may not have $\operatorname{cl}\left(A \cup B_{1}\right)-B_{1} \subseteq E-B$. We get pseudomodularity of strict gammoids as a corollary.

Corollary 3.7. Let $M$ be a strict gammoid, then $M$ is pseudomodular.

The converse of Theorem 3.6 is not true. We show the following example of a pseudomodular gammoid that is not 3 -flat.

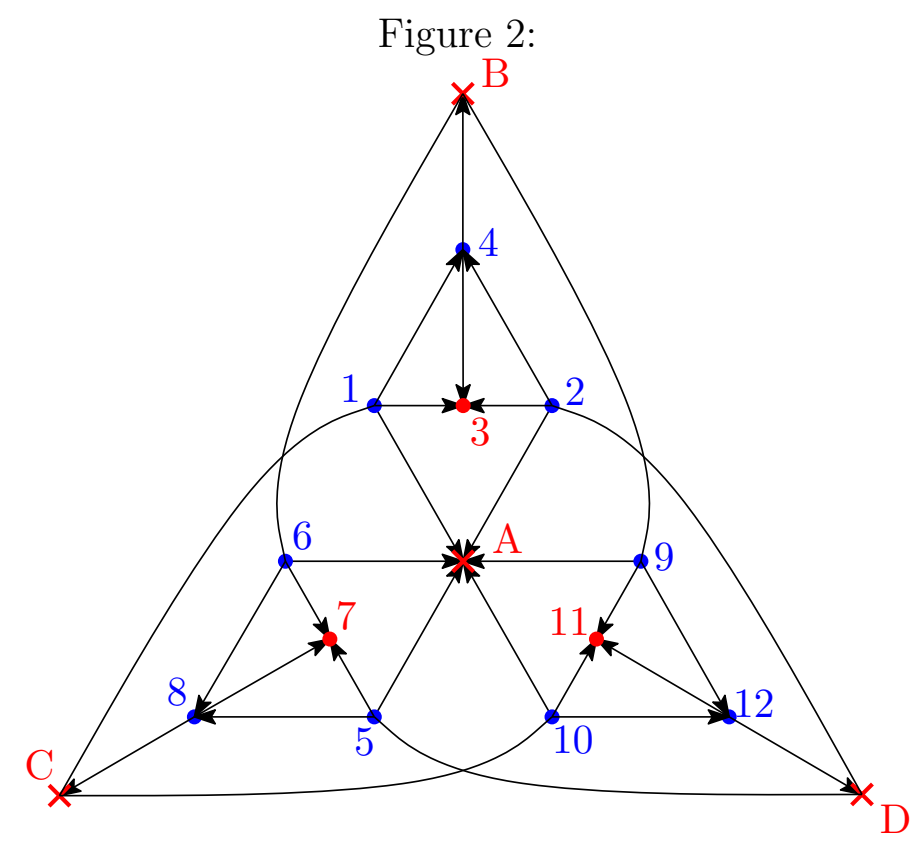


Example 2. Let $N=\{1,2,3,4,5,6,7,8,9,10,11,12\}$ and let $M$ be the matroid on $N$ which has as flats any subset of $N$ of size less or equal to $4,\{1,3,4,6,7,8\},\{2,3,4,9,11$, $12\},\{5,7,8,10,11,12\}$, any subset of size 5 not contained in any of the previous 3 , $F_{1}=\{1,2,3,4,5,6,7,8\}, F_{2}=\{1,2,3,4,9,10,11,12\}, F_{3}=\{5,6,7,8,9,10,11,12\}$ and $N$. Figure 2 shows a strict gammoid, with sinks $A, B, C, D, 3,7,11$ where restricting $\{A, B, C, D\}$ gives us the matroid $M$.

Note that if $\mathcal{C}=\left\{F_{1}, F_{2}, F_{3}\right\}$, we have $F_{\{1,2\}}=\{1,2,3,4\}, F_{\{1,3\}}=\{5,6,7,8\}, F_{\{2,3\}}=$ $\{9,10,11,12\}$ and $F_{\{1,2,3\}}=\emptyset$. So $\Delta(\mathcal{C})=7-3 \cdot 6+3 \cdot 4=1$, so $M$ is not 3-flat. Note that for any flat $B$ which is independent we have $A \triangleleft B$ for every $A \in \mathcal{F}$, as $B_{0}$ would be the set of elements in $B$ that are not coloop in $B \cup A$. So the only flats we need to check are $F_{1}, F_{2}, F_{3},\{1,3,4,6,7,8\},\{2,3,4,9,11,12\}$ and $\{5,7,8,10,11,12\}$, which are easy to do using Lemma 3.5. This is our first example of a pseudomodular gammoid which is not a strict gammoid.

\section{Flatness Degree}

As we have seen being 3-flat implies many interesting properties such as pseudomodularity. It may be worth noting that 3-flatness is sufficient for CM-triviality in [8]. This fact got us interested in studying $n$-flatness in general. We can begin with the following natural definition

Definition 4.1. Given a matroid $M$ we say that $\phi(M)$ is the flatness degree of $M$ if $M$ is $\phi(M)$-flat but not $(\phi(M)+1)$-flat. If such an integer does not exist (i. e. $M$ is totally flat), $\phi(M)=\omega$

As $n$ increases it becomes much harder to study $n$-flatness. However, we will show that for each $n$ there is a matroid with flatness degree $n$, in other words, being $n$-flat is in fact different for each $n$. First we start by presenting some tools that are useful for studying the $\Delta$ function. Proposition 4.2 was already shown by Holland in [7], which includes a much deeper study of the $\Delta$ function (there called $T$ function). Let $\mathcal{C}=\left\{F_{i} \in \mathcal{F} \mid k \in I\right\}$ be a collection of flats of any matroid.

Proposition 4.2. Suppose that there are $F_{i}, F_{j} \in \mathcal{C}$ such that $F_{i} \subseteq F_{j}$. Then $\Delta(C)=$ $\Delta\left(C-\left\{F_{i}\right\}\right)$

Proof. Note that $\bigcup C=\bigcup\left(C-\left\{F_{i}\right\}\right)$. Let $\mathcal{C}_{i}=\left\{F_{\{i, k\}} \mid k \in I \wedge i \neq k\right\}$. So $\Delta(C)=$ $\Delta\left(C-\left\{F_{i}\right\}\right)-\Delta\left(\mathcal{C}_{i}\right)$. But for each $S \subseteq I-\{i, j\}, F_{S \cup\{i\}}=F_{S \cup\{i, j\}}$ so $\Delta\left(\mathcal{C}_{i}\right)=0$ and we have the desired result.

Proposition 4.3. There exists $\mathcal{C}^{\prime}$ such that $\left|\mathcal{C}^{\prime}\right|=|\mathcal{C}|, \Delta\left(\mathcal{C}^{\prime}\right) \geqslant \Delta(\mathcal{C})$ and all elements of $\mathcal{C}^{\prime}$ are cyclic.

Although this proposition was not previously stated as it is, it is widely regarded that being transversal (as well as being a strict gammoid and hence by being totally flat) depends only on the cyclic flats. So the proposition is natural. 
Proof. The proof is done by induction on the sum of the number of isthmuses of the elements in $\mathcal{C}$. If the sum is 0 we have the desired result. Now suppose $F_{i} \in \mathcal{C}$ has an isthmus $e$. Let $S:=\left\{i \in I \mid e \in F_{i}\right\}$. First suppose $e$ is also an isthmus in $F_{\emptyset}$. Then every subset of $F_{\emptyset}$ who contains $e$, has it as an isthmus. Now for every $j \in S, F_{j}-\{e\}$ is also a flat different than $F_{k}$ for every $k \neq j$. Consider $\mathcal{D}:=\left\{F_{j} \mid j \notin S\right\} \cup\left\{F_{k}-\{e\} \mid k \in S\right\}$. We have $|\mathcal{D}|=|\mathcal{C}|$, and $\Delta(\mathcal{C})-\Delta(\mathcal{D})=\sum_{A \subseteq S}(-1)^{|A|}=0$. As $\mathcal{D}$ has $|S|$ less isthmuses than $\mathcal{C}$ by induction hypothesis there is a collection of cyclic flats $\mathcal{C}^{\prime}$ such that $\left|\mathcal{C}^{\prime}\right|=|\mathcal{D}|=|\mathcal{C}|$ and $\Delta\left(\mathcal{C}^{\prime}\right) \geqslant \Delta(\mathcal{D})=\Delta(\mathcal{C})$

Now suppose $e$ is not an isthmus in $F_{\emptyset}$. Let $\mathcal{D}:=\left\{F_{j} \mid j \neq i\right\} \cup\left\{F_{i}-\{e\}\right\}$. Again $F_{i}-\{e\} \neq F_{j}$ for $i \neq j$ and $|\mathcal{D}|=|\mathcal{C}|$. If $|S| \geqslant 2$, have $\Delta(\mathcal{C})-\Delta(\mathcal{D})=\sum_{A \subseteq(S-\{i\})}(-1)^{|A|+1}=$ 0 . If $S=\{i\}$ then $\Delta(\mathcal{C})-\Delta(\mathcal{D})=-1$. The number of isthmuses in $\mathcal{D}$ is one less than in $\mathcal{C}$ so by the induction hypothesis the proposition such $\mathcal{C}^{\prime}$ exists.

Proposition 4.4. Suppose that $|\mathcal{C}|>1$. Then there exists $\mathcal{C}^{\prime}$ such that $\left|\mathcal{C}^{\prime}\right|=|\mathcal{C}|, \Delta\left(\mathcal{C}^{\prime}\right) \geqslant$ $\Delta(\mathcal{C})$ and $\forall F \in \mathcal{C}^{\prime} F \subseteq c l\left(\bigcup\left(\mathcal{C}^{\prime}-\{F\}\right)\right)$

Proof. Let $s(\mathcal{C})=\sum_{i \in I} r\left(F_{i}-c l\left(\bigcup_{j \neq i} F_{j}\right)\right)$. If $s(\mathcal{C})=0$, then clearly $\forall i \in I \quad F_{i} \subseteq c l\left(\bigcup_{j \neq i} F_{j}\right)$. Now we will proceed by induction on $s(\mathcal{C})$. Suppose $s(\mathcal{C})>0$. Then there exists $i \in I$ such that $r\left(F_{i}-\operatorname{cl}\left(\bigcup_{j \neq i} F_{j}\right)\right) \geqslant 1$. Let $x \in F_{i}-c l\left(\bigcup_{j \neq i} F_{j}\right)$ non loop, and take any $j \in I j \neq i$. Consider $F_{j}^{\prime}=\operatorname{cl}\left(F_{j} \cup\{x\}\right)$ and $\mathcal{D}=\left\{F_{k} \mid k \in I \quad k \neq j\right\} \cup\left\{F_{j}^{\prime}\right\}$. Clearly $|\mathcal{C}|=|\mathcal{D}|$. Note that if $A \subseteq I-\{i, j\}$ we have $F_{j}^{\prime} \cap F_{A}=F_{A \cup\{j\}}$. As $x \in\left(F_{j}^{\prime} \cap F_{i}\right)-\left(F_{j} \cap F_{i}\right)$ we have

$$
\begin{aligned}
\Delta(\mathcal{D})-\Delta(\mathcal{C}) & =-r\left(F_{j}^{\prime}\right)+r\left(F_{j}^{\prime} \cap F_{i}\right)+r\left(F_{j}\right)-r\left(F_{j} \cap F_{i}\right) \\
& =r\left(F_{j}^{\prime} \cap F_{i}\right)-r\left(F_{j} \cap F_{i}\right)+1 \\
& \geqslant 0 .
\end{aligned}
$$

But now $r\left(F_{i}-\operatorname{cl}\left(\bigcup_{k \neq i, j} F_{k} \cup\left\{F_{j}^{\prime}\right\}\right)\right)<r\left(F_{i}-\operatorname{cl}\left(\bigcup_{k \neq i} F_{k}\right)\right)$, so $s(\mathcal{D})<s(\mathcal{C})$. Then by the induction hypothesis, there is a $\mathcal{C}^{\prime}$ such that $\left|\mathcal{C}^{\prime}\right|=|\mathcal{D}|$ and $\Delta\left(\mathcal{C}^{\prime}\right) \geqslant \Delta(\mathcal{D}) \geqslant \Delta(\mathcal{C})$ and $\forall F \in \mathcal{C}^{\prime} F \subseteq \operatorname{cl}\left(\bigcup\left(\mathcal{C}^{\prime}-\{F\}\right)\right)$.

It is now easy to verify that one can assume that all elements of $\mathcal{C}$ must be of rank at least 2. So we can focus on studying only the particular class of collections of flats which are cyclic, with rank at least 2, no flat is contained in another, and every flat is contained in the closure of the union of the rest of the flats. Before we exhibit matroids with fixed flatness degree, we prove the following combinatorial lemma.

Lemma 4.5. Let $n$ be a positive integer, and $l, m$ positive integers less than $n$. Then

$$
\sum_{k=0}^{m}(-1)^{k}\left(\begin{array}{c}
n-k \\
l
\end{array}\right)\left(\begin{array}{c}
m \\
k
\end{array}\right)=\left(\begin{array}{c}
n-m \\
l-m
\end{array}\right) .
$$

In particular, when $m>l$ the equation is equal to 0 . 
Proof. Note that the $\left(\begin{array}{c}n-k \\ l\end{array}\right)\left(\begin{array}{c}m \\ k\end{array}\right)$ is the number of ways of choosing a subset $K$ of $[m]$ of cardinality $k$ and then choosing a subset $L$ of $[n]$ of cardinality $l$ such that $L \cap K=\emptyset$. The LHS is summing the number of ways to take an arbitrary subset $K \subseteq[\mathrm{m}]$ and then choosing $L$ with an alternating sign depending on $|K|$. Now if we choose first the set $L$, we have now to choose subsets $K$ of $[m]-L$. So for a fixed set $L$ where $j=|[m]-L|$ we will have a contribution of $\sum_{k=0}^{j}\left(\begin{array}{l}j \\ k\end{array}\right)(-1)^{j}$. Now this is 0 for $j \geqslant 1$ and 1 for $j=0$. So the only terms that survive are the ones where $[m] \subseteq L$. But the number of ways of choosing $L$ such that $[m] \subseteq L \subseteq[n]$ is precisely $\left(\begin{array}{c}n-m \\ l-m\end{array}\right)$

Now we have the tools to prove the following theorem

Theorem 4.6. Let $n \geqslant 2$. Then there exists a matroid $M$ with flatness degree $n$. Moreover, there exists a gammoid with flatness degree $n$.

Proof. First we assume $n \geqslant 5$ and we construct a gammoid such that $\phi(M)=n-1$. Consider $N:=\left(\begin{array}{c}{[n]} \\ 2\end{array}\right)$, the subsets of $[n]:=\{1,2, \ldots n\}$ of size 2. For $i \in[n]$ let $A_{i}=\{x \in$ $N \mid i \in x\}$. Consider the transversal matroid $M$ on the set $N$ given by the presentation $\left(A_{1}, A_{2}, \ldots, A_{n}, N, N \ldots N\right)$, where there are $\left(\begin{array}{c}n-1 \\ 2\end{array}\right)-n$ copies of $N$ in $\mathcal{A}$. Note that as $M$ is transversal, it is a gammoid.

For $i \in[n]$ let $F_{i}:=\{x \in N \mid i \notin x\}$. We claim that these are the only non trivial cyclic flats in the matroid. Suppose $D \subseteq N$ is dependent. As there are $\left(\begin{array}{c}n-1 \\ 2\end{array}\right)-n$ copies of $N$ in the presentation of $M$, we have that $|D|>\left(\begin{array}{c}n-1 \\ 2\end{array}\right)-n$. But now for $n \geqslant 5$ we have that $\left(\begin{array}{c}n-1 \\ 2\end{array}\right)-n \geqslant\left(\begin{array}{c}n-3 \\ 2\end{array}\right)$. So $D$ would have access to $A_{i}$ for at least $n-2$ different $i$ 's in $[n]$. But then we would have that $|D| \geqslant\left(\begin{array}{c}n-1 \\ 2\end{array}\right)-1$. But $\left(\begin{array}{c}n-1 \\ 2\end{array}\right)-1>\left(\begin{array}{c}n-2 \\ 2\end{array}\right)$, so a subset this big would necessarily have access to $A_{i}$ for at least $n-1$ different $i$ 's in $[n]$. So $|D|>\left(\begin{array}{c}n-1 \\ 2\end{array}\right)$ and $r(D) \geqslant r(M)-1$. So the only non trivial flats that can be cyclic are the hyperplanes which are precisely the sets $F_{i}$. We could have more easily defined $M$ by giving $\left\{F_{i} \mid i \in[n]\right\}$ as its set of hyperplanes, but we wanted to show that it is in fact a gammoid.

So to check that $M$ is $k$-flat for a given $k$, by proposition 4.3 we need only to focus on collections of $F_{i}$ as they are the only non trivial cyclic flats of $M$. Let $A \subseteq[n]$ of size $m>2$. Consider $\mathcal{C}:=\left\{F_{i} \mid i \in A\right\}$. If $B \subseteq A$ with $|B|>2$, then we have that $F_{B}=$ $\{x \in N \mid x \cap B=\emptyset\}$. So $F_{B}$ is independent and $r\left(F_{B}\right)=\left|F_{B}\right|=\left(\begin{array}{c}n-|B| \\ 2\end{array}\right)$. As $F_{i}$ is a 
circuit for every $i \in[n]$, we have $r\left(F_{i}\right)=\left(\begin{array}{c}n-1 \\ 2\end{array}\right)-1$. Finally, $r\left(F_{\emptyset}\right)=r(N)=\left(\begin{array}{c}n-1 \\ 2\end{array}\right)$. So using lemma 4.5 we have

$$
\begin{aligned}
\Delta(\mathcal{C}) & =\sum_{k=2}^{m}(-1)^{k}\left(\begin{array}{c}
n-k \\
2
\end{array}\right)\left(\begin{array}{l}
m \\
k
\end{array}\right)-m\left(\left(\begin{array}{c}
n-1 \\
2
\end{array}\right)-1\right)+\left(\begin{array}{c}
n-1 \\
2
\end{array}\right) \\
& =\sum_{k=0}^{m}(-1)^{k}\left(\begin{array}{c}
n-k \\
2
\end{array}\right)\left(\begin{array}{l}
m \\
k
\end{array}\right)+m+\left(\begin{array}{c}
n-1 \\
2
\end{array}\right)-\left(\begin{array}{c}
n \\
2
\end{array}\right) \\
& =m+\left(\begin{array}{c}
n-1 \\
2
\end{array}\right)-\left(\begin{array}{l}
n \\
2
\end{array}\right) \\
& =m-n+1 .
\end{aligned}
$$

So $\Delta(\mathcal{C})>0$ if and only if $m=n$. So $\phi(M)=n-1$. So we have constructed gammoids such that $\phi(M)$ is any integer greater or equal to 4. Any strict gammoid has flatness degree $w$ and example 1 shows a gammoid with flatness degree 2. So the only possible flatness degree which we have not yet exhibited a matroid with said degree is 3. As stated above, the same matroid structure of the last construction can be defined by its hyperplanes and can be used to construct a matroid of flatness degree 3 . However this construction would be the matroid generated by graph $K_{4}$, which Mason proved in [13] not to be a gammoid. However example 2 in [13] is an example of a gammoid with flatness degree 3 .

Having differentiated all of this properties we see now that this is an infinite chain of successively stronger properties

$$
\text { Pseudomodular } \supset \text { 3-Flat } \supset \text { 4-Flat } \supset \cdots \supset \text { Totally Flat }
$$

All of the inclusions are strict, even in the class of gammoids, with the examples shown above. Note that all of the properties are conditions on the lattice of flats. Modularity, which is also a condition on the lattice of flats, implies 3-flatness by Proposition 4.4. However, it does not imply 4 -flatness (take 4 planes in $\mathbb{R}^{3}$ which intersect in different lines).

\section{Problems}

A full algebraic matroid consists of an algebraically closed field $K$ where the rank function is the transcendence degree over a subfield $F$ of $K$. As algebraically closed fields are infinite, full algebraic matroids are infinite matroids. However in [4] is proved they are pseudomodular as generalization from the lemma Ingleton and Main used in [10] when they first constructed a non-algebraic matroid. As pseudomodularity is the first step towards 3-flatness, the following becomes a natural question:

Problem 1. What is the minimum possible flatness degree for a full algebraic matroid? 
If a matroid $M$ has flatness degree $n$ for a large finite $n$, it seems that $M$ must have necessarily a large rank. On the other hand, for rank 3 matroids flatness degree 3 is possible $\left(K_{4}\right)$ but not flatness degree 2 . However, as there are no non strict gammoids of rank 3 [11], the only possible flatness degree for a gammoid of rank 3 is $\omega$. So the bounds for flatness degree for gammoids and matroids in general are different.

Problem 2. For a given integer $n>1$, what is the minimum possible rank for a matroid $M$ such that $\phi(M)=n$ ?. What is the minimum possible rank for a gammoid $M$ such that $\phi(M)=n$ ?

The fact that these minimum ranks are different may help the widely known open problem $[5,14]$

Problem 3. Is there an algorithm to determine whether or not a given matroid is a gammoid?

\section{Acknowledgements}

The author would like to thank Alf Onshuus: without his valuable comments and guidance this article would not have been possible.

\section{References}

[1] A Björner and L Lovász. Pseudomodular lattices and continuous matroids. Acta Scientarium Mathematicarum 51:295-308, 1987.

[2] Richard A Brualdi. Introduction to matching theory. Combinatorial Geometries, 29:53, 1987.

[3] Richard A Brualdi. Transversal matroids. Combinatorial Geometries, 29:72-97, 1987.

[4] A Dress and L Lovász. On some combinatorial properties of algebraic matroids. Combinatorica, 7(1):39-48, 1987.

[5] David M Evans. Matroid theory and Hrushovski's predimension construction. Preprint, 2011. arXiv:1105.3822

[6] Winfried Hochstättler and Walter Kern. Matroid matching in pseudomodular lattices. Combinatorica, 9(2):145-152, 1989.

[7] KL Holland. Flatness, convexity and notions of freeness in combinatorial geometries. algebra universalis, 41(1):1-21, 1999.

[8] Ehud Hrushovski. A new strongly minimal set. Annals of Pure and Applied Logic, 62(2):147-166, 1993.

[9] AW Ingleton. Transversal matroids and related structures. In Higher Combinatorics, pages 117-131. Springer, 1977.

[10] AW Ingleton and RA Main. Non-algebraic matroids exist. Bulletin of the London Mathematical Society, 7(2):144-146, 1975. 
[11] AW Ingleton and MJ Piff. Gammoids and transversal matroids. Journal of Combinatorial Theory, Series B, 15(1):51-68, 1973.

[12] JH Mason. A characterization of transversal independence spaces. In Théorie des matrö̈des, pages 86-94. Springer, 1971.

[13] JH Mason. On a class of matroids arising from paths in graphs. Proceedings of the London Mathematical Society, 3(1):55-74, 1972.

[14] James G Oxley. Matroid theory. Oxford University press, 1992. 